\title{
Terapia vestibular en vestibulopatía bilateral por gentamicina: Reporte de un caso y revisión de la literatura
}

\author{
Vestibular therapy in a case of gentamicin-induced vestibulopathy: \\ Case report and literature revision
}

\author{
Patricia Oyarzún D¹, Graciela Gómez M¹, Carolina Valera C², Sergio Jiménez C¹.
}

\begin{abstract}
RESUMEN
La vestibulopatía bilateral es poco frecuente, se caracteriza principalmente por inestabilidad al caminar o al estar de pie, visión borrosa inducida por el movimiento u oscilopsia al caminar o al realizar movimientos rápidos de la cabeza o del cuerpo, empeoramiento de la estabilidad en la oscuridad o terrenos irregulares, reducción de los síntomas al estar en condiciones estáticas, ganancia del reflejo vestíbulo-ocular angular reducida de forma bilateral, entre otros. Existen múltiples causas. Dentro de las causas identificables, se describen principalmente medicamentos ototóxicos, meningitis y enfermedad de Ménière. Se presenta el caso de una paciente de 64 años diagnosticada con vestibulopatía bilateral posterior a tratamiento intramuscular con gentamicina por sobreinfección bacteriana cutánea de las manos. La evaluación vestibular complementada con videonistagmografía y prueba de impulso cefálico asistida por video confirman el diagnóstico y se inicia tratamiento con rehabilitación vestibular enfocada en promover la compensación central a través de estrategias de sustitución principalmente; además de habituación y adaptación vestibular, favoreciendo la estabilización de la mirada, mantención del equilibrio, control postural, marcha y reducción de los síntomas.

Palabras clave: Vestibulopatía bilateral, gentamicina, ototoxicidad, oscilopsia.
\end{abstract}

\begin{abstract}
Bilateral vestibulopathy is infrequent, and it is characterized mostly by unstable walking or when standing, blurred vision induced by movement, or oscillopsia when walking or performing fast movements; worsening of the stability in darkness or uneven ground, but with lack of symptoms in static conditions. Other symptoms may include bilateral reduction of the oculo-vestibular reflex. Among the identifiable causes, there is the use of ototoxic medication, meningitis, Ménière's disease, although it can be idiopathic or have a neurological cause. We hereby describe the case of a 64-year-old woman, diagnosed with bilateral vestibulopathy secondary to intramuscular treatment with gentamicin due to a bacterial hand infection. Vestibular assessment was complemented with video-nystagmography and video head impulse test which confirmed the diagnosis, and therapy was started with vestibular rehabilitation focused on promoting central compensation mainly, through substitution strategies. Also, habituation exercise and vestibular adaptation strategies were used, thus promoting sight stabilization, balance maintenance, postural control, walking, and reduction of the symptoms.
\end{abstract}

Key words: Bilateral vestibulopathy, gentamicin, ototoxicity, oscillopsia.

Escuela de Fonoaudiología. Facultad de Salud. Universidad Santo Tomás, Chile.

2 Servicio de Otorrinolaringología. Hospital Regional de Talca, Chile.

Los autores declaran no presentar conflicto de interés.

Recibido el 27 de agosto de 2019. Aceptado el 16 de octubre de 2019. 


\section{INTRODUCCIÓN}

La vestibulopatía bilateral (BVP) afecta aproximadamente a 28 de cada 100.000 adultos según reportes de una encuesta nacional de salud en EE.UU1'. Considerando el historial del paciente, el examen físico y los exámenes solicitados, la Sociedad de Bárány ha establecido los criterios para su correcto diagnóstico en el año 2017, los que consideran "inestabilidad al caminar 0 estar de pie al menos uno o dos minutos, visión borrosa inducida por el movimiento u oscilopsia al caminar o movimiento de cabeza y/o cuello, empeoramiento de la inestabilidad en la oscuridad o terrenos irregulares, ausencia de síntomas al estar sentado 0 acostado en condiciones estáticas, ganancia del reflejo vestíbulo-ocular (RV0) angular reducida 0 ausente en forma bilateral y no ser explicada mejor por otra enfermedad"2.

Respecto a la etiología de la BVP se han descrito múltiples causas. Dentro de las causas identificables, destacan principalmente la enfermedad de Ménière, ototoxicidad y meningitis. La ototoxicidad puede ocurrir luego del uso de fármacos como la gentamicina y otros antibióticos aminoglucócidos, diuréticos de asa, quimioterapia con agentes platinados, estirenos, y varios más ${ }^{3-9}$. Otras causas son alteraciones genéticas, enfermedades autoinmunes, tumores como schwannoma vestibular bilateral, carcinomatosis meníngea, entre otros ${ }^{10}$. Sin embargo, existe un importante número de casos en donde se ha establecido una causa idiopática o neurodegenerativa ${ }^{11,12}$. Respecto al tratamiento, actualmente existe evidencia suficiente que respalda la terapia vestibular como tratamiento en pacientes con BVP ya que ofrece beneficios claros y sustanciales ${ }^{13-15}$, mejorando de esta forma la estabilidad de la mirada, el equilibrio y la marcha.

\section{CASO CLÍNICO}

Se describe el caso de una paciente de 64 años de edad con antecedentes mórbidos de hipertensión arterial y diabetes mellitus tipo 2, que recibió tratamiento con gentamicina intramuscular durante 7 días (80 mg/día) por sobreinfección bacteriana cutánea de las manos, la cual anteriormente había sido tratada con otras terapias antibióticas no ototóxicas sin resultados favorables. Posterior al tratamiento médico con gentamicina, refiere mareo, náuseas y vómitos; además de la manifestación progresiva de desequilibrio intenso, inestabilidad en la marcha y oscilopsia; con una duración variable de horas a días por lo que decide consultar a otorrinolaringólogo. Al examen clínico, se evidencia una prueba de impulso cefálico clínico positivo bilateral, ausencia de nistagmo espontáneo, pruebas cerebelosas normales, dificultad para realizar pruebas de equilibrio estático y dinámico por marcada inestabilidad y pulsión hacia posterior, prueba de Dix-Hallpike negativa, Weber indiferenciado y Rinne positivo bilateral, además de otoscopía normal. Se complementa el estudio con audiometría, videonistagmografía (VNG) computarizada con estímulos calóricos y prueba de impulso cefálico asistida por video (V-HIT). La audiometría evidencia hipoacusia sensorioneural bilateral de grado leve (Figura 1), con 96\% de discriminación en oído derecho

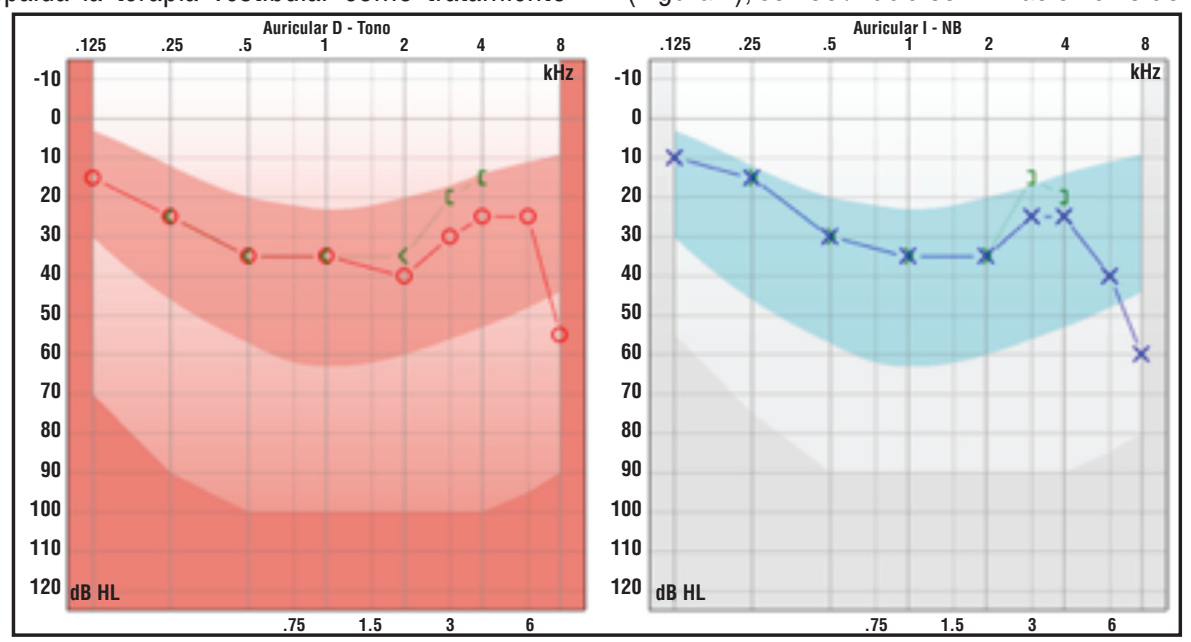

Figura 1. Audiometría tonal. Hipoacusia sensorioneural con promedio tonal puro (PTP) OD vía área $36.6 \mathrm{~dB}$ y vía ósea $35 \mathrm{~dB}$; Ol vía aérea $33.3 \mathrm{~dB}$ y vía ósea $33.3 \mathrm{~dB}$. 


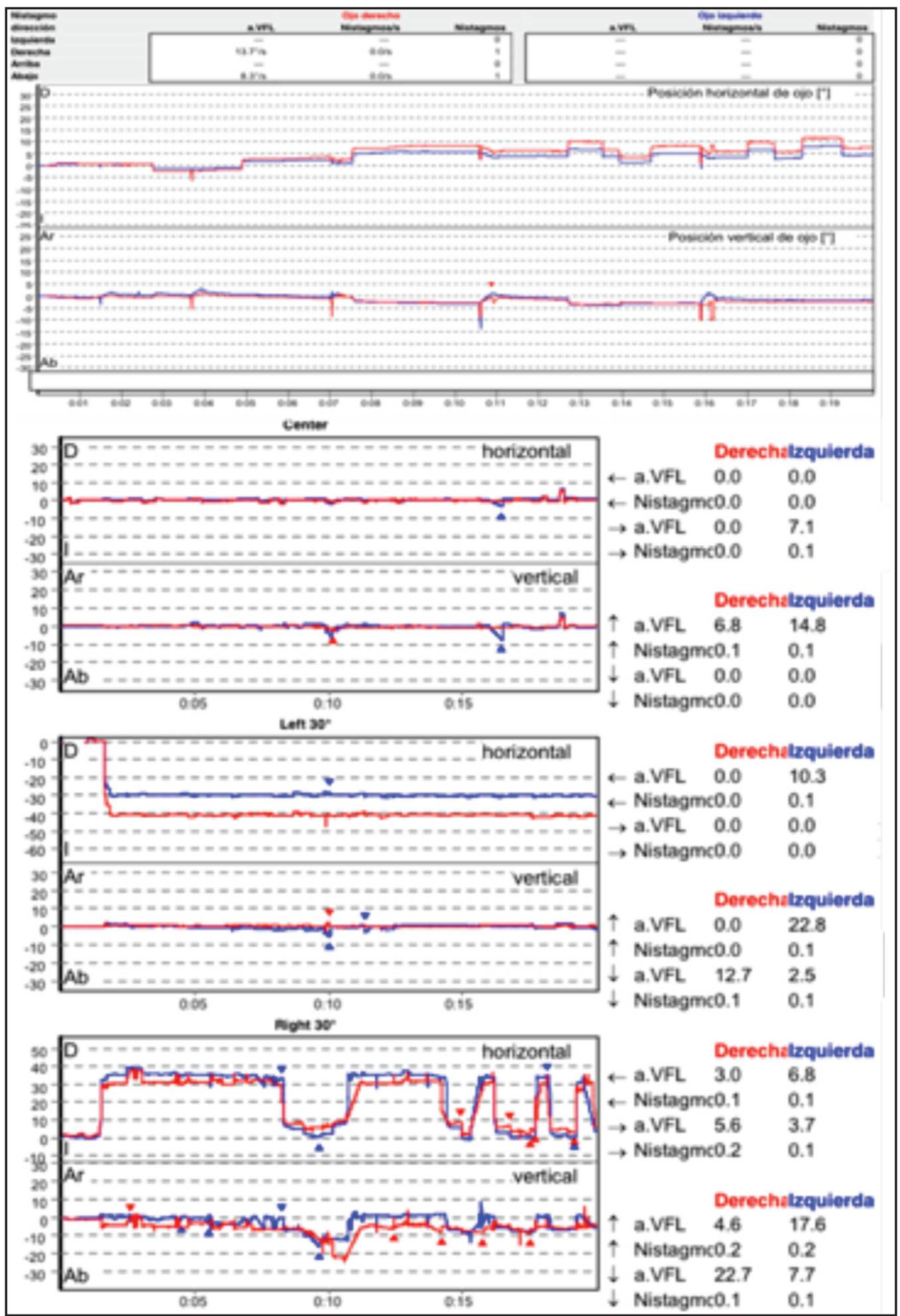

Figura 2. Estudio del nistagmo espontáneo sin fijación y con fijación ocular en mirada central, izquierda y derecha. 
y $96 \%$ en oído izquierdo para estímulos bisílabos. En la VNG, se aprecia ausencia de nistagmo espontáneo con y sin fijación ocular (Figura 2), prueba calórica con aire arroja hipofunción vestibular bilateral, con una velocidad de fase lenta (VFL) total de $6,9 \%$ s e índice de fijación de $0 \%$ (Figura 3).
La prueba de V-HIT revela ganancia del RVO disminuida de forma bilateral con 0,02 para derecha e izquierda en el plano horizontal, presencia de sacadas covert y overt bilaterales; y porcentaje de asimetría 0\% (Figura 4). Además, se aprecian oscilaciones constantes con aumento de la base

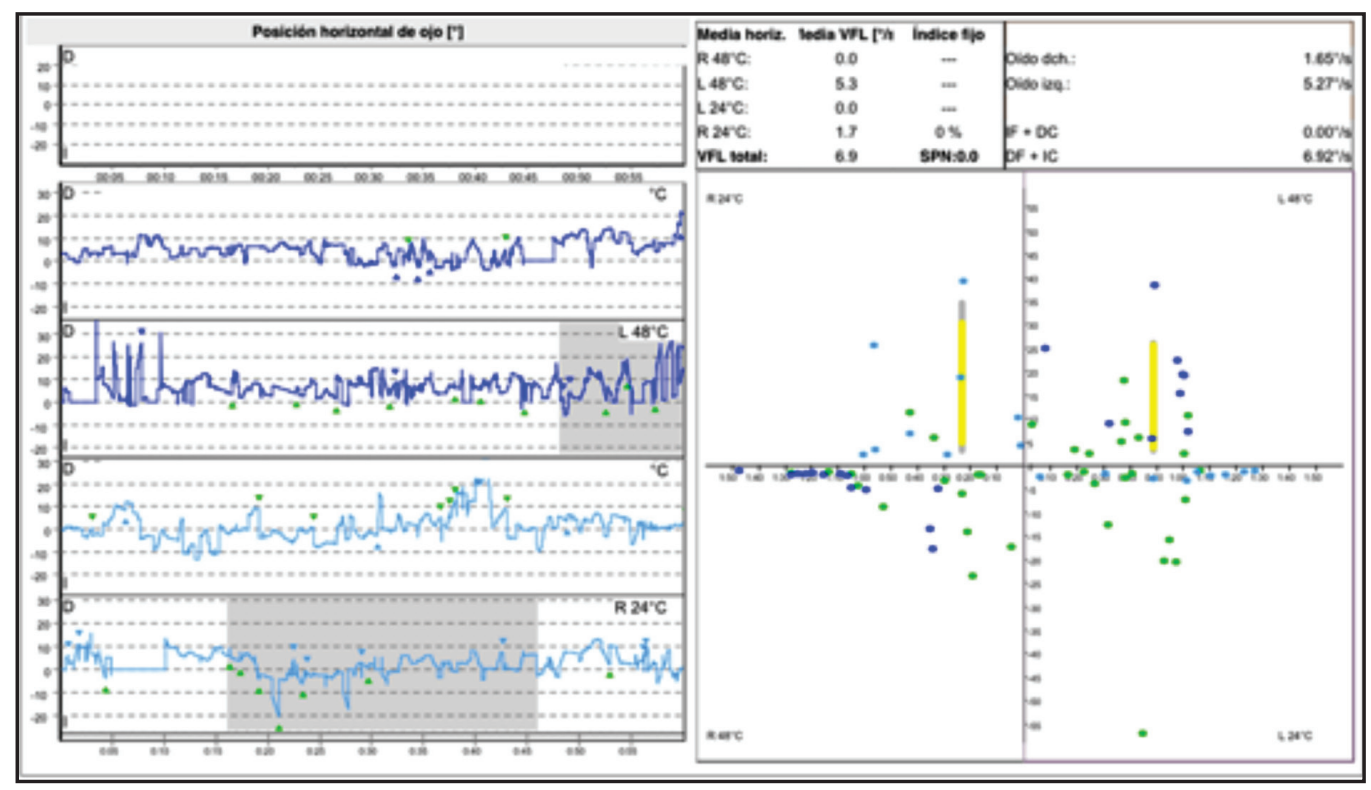

Figura 3. Prueba calórica con aire $24^{\circ} \mathrm{C}$ frío y $48^{\circ} \mathrm{C}$ calor. Hipofunción vestibular bilateral.

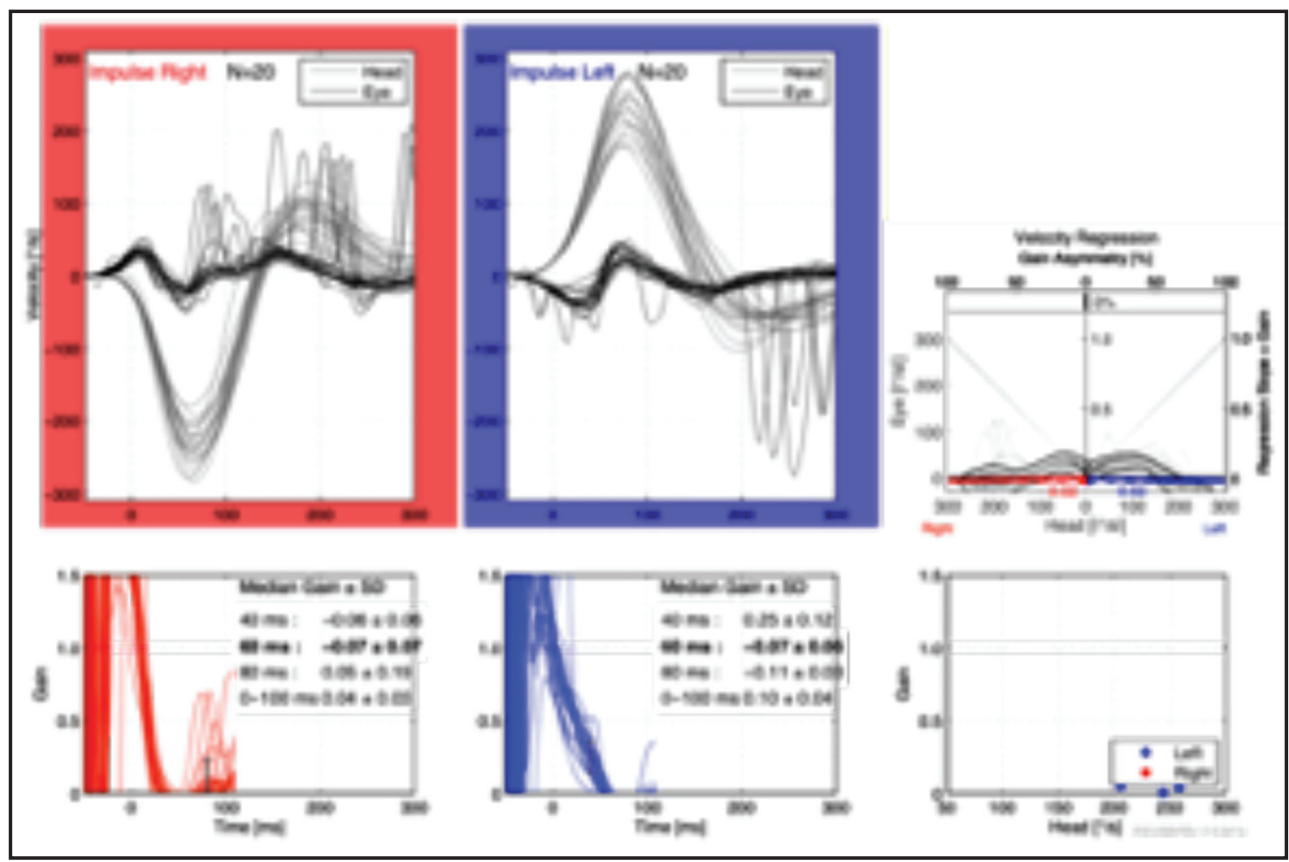

Figura 4: V-HIT inicial de plano horizontal. Ganancia disminuida bilateralmente. Presencia de sacadas covert y overt. 
de sustentación e imposibilidad para realizar marcha.

La paciente es derivada a rehabilitación vestibular (RV). En la evaluación inicial, se evidencia discapacidad severa en los aspectos emocional, funcional y físico de acuerdo al test de discapacidad vestibular dizziness handicap inventory (DHI), agudeza visual dinámica alterada según la prueba dynamic visual acuity test (DVA), alcance funcional y apoyo monopodal alterado obtenido a través de la prueba functional reach test (FRT) y test de apoyo monopodal, respectivamente. Las pruebas de marcha según tiempo y modalidad (normal, con cambios de velocidad y con movimientos de cabeza) se vieron notoriamente alteradas según timed up and go (TUG) y dynamic gait index (DGI).

El programa de RV incluyó diez sesiones de intervención con una duración de 60 minutos cada una. Se programó una reevaluación intermedia posterior a la quinta sesión y una reevaluación final una vez concluidas las diez sesiones, las cuales se organizaron dos veces por semana durante las tres primeras semanas y las cuatro sesiones restantes fueron programadas una vez a la semana. Los ejercicios estuvieron enfocados en desarrollar y utilizar estrategias compensatorias que incluyen principalmente la sustitución del mecanismo deficiente por información visual y/o somatosensorial para mejorar el control postural, la mantención del equilibrio y la marcha. Asimismo, se realizaron ejercicios para estabilizar la mirada, aumentar la ganancia del reflejo cérvico-ocular y reducir los síntomas asociados. Se reforzaron en el hogar los aspectos abordados durante la sesión, con una frecuencia de 3 veces al día y una duración de 30 minutos cada una.

Específicamente, las sesiones de intervención se iniciaron con ejercicios de marcha lineal sobre superficie regular, incorporando obstáculos sim- ples (10-15 cm de alto) desde la primera sesión, que requerían que la paciente realizara flexión de cadera y rodilla. El apoyo brindado consistía en una pared ubicada lateralmente a la dirección de desplazamiento de manera que pudiera apoyarse cuando fuera necesario; además del acompañamiento por parte del terapeuta. Asimismo, se incorporaron ejercicios de seguimiento lento en plano horizontal con movimiento cefálico y flexiónextensión cervical con fijación ocular. Desde la tercera sesión, se incluye mayor complejidad en cuanto al aumento de obstáculos y uso de superficies irregulares e inestables durante la marcha. Respecto a los ejercicios cervicales se incrementa la complejidad realizando cambio de posición de pie a sentado en superficie inestable con fijación ocular y estímulo móvil proyectado en pantalla de televisión. Cabe destacar que el progreso en las primeras sesiones fue difícil debido al afectado componente emocional de la paciente por su condición actual evidenciado en el cuestionario DHI (Tabla 1), aunque los avances en aspectos de marcha y equilibrio ya eran notorios. De acuerdo a esto, se reeduca sobre los beneficios e importancia de la RV y se sugiere derivación a psicoterapia, ante lo cual la paciente se niega.

Las sesiones realizadas posteriores a la evaluación intermedia, mostraron un progreso significativo, lo que permitió el avance de los ejercicios en circuito de marcha con obstáculos, apoyo monopodal, superficies inestables, ascenso-descenso, equilibro estático incorporado y ejercicios de movimiento cervical en plano horizontal y vertical con fijación ocular en marcha. Para concluir el programa, se realizaron dos sesiones enfocadas en el uso de realidad virtual por medio de videos en formato "rv" (realidad virtual) visualizados a través de dispositivo móvil de realidad virtual, iniciando los ejercicios solo con movimientos

Tabla 1: Resultados dizzines handicap inventory (DHI)

\begin{tabular}{|lcccccc|}
\hline & \multicolumn{2}{c}{ Evaluación previa } & \multicolumn{2}{c|}{ Evaluación intermedia } & \multicolumn{2}{c|}{ Evaluación final } \\
\hline Aspecto & Puntaje & Categoría & Puntaje & Categoría & Puntaje & Categoría \\
\hline Físico & 22 & Severo & 14 & Moderado & 8 & Normal \\
Funcional & 32 & Severo & 32 & Severo & 24 & Moderado \\
Emocional & 32 & Severo & 32 & Severo & 22 & Moderado \\
\hline
\end{tabular}


cefálicos e incorporando marcha para finalizar. La reevaluación consideró los protocolos aplicados durante la evaluación inicial en relación al cuestionario DHI (Tabla 1), equilibrio (Tabla 2) y V-HIT de plano horizontal (Figuras 5 y 6). La evolución fue favorable en la mayor parte de los aspectos abordados.

En DHI, se evidenció un avance significativo en el aspecto físico, pues comienza la terapia en una situación de "discapacidad severa" y culmina en categoría de "sin discapacidad". Sin embargo, el progreso en los aspectos funcional y emocional fue más restringido, especialmente entre la evaluación previa e intermedia. Si bien, posterior a las diez sesiones se aprecia una "discapacidad moderada", consideramos que la derivación a psicoterapia es relevante para el caso, ya que es el principal aspecto que se mantuvo afectado posterior a la RV. Respecto a DVA, se observa una alterada capacidad de discriminar un objeto al incorporar movimiento cefálico producto de la visión borrosa referida

Tabla 2: Resultados evaluación del equilibrio

\begin{tabular}{|c|c|c|c|c|c|c|}
\hline \multirow[b]{2}{*}{ Test } & \multicolumn{2}{|c|}{ Evaluación previa } & \multicolumn{2}{|c|}{ Evaluación intermedia } & \multicolumn{2}{|c|}{ Evaluación final } \\
\hline & Puntaje & Categoría & Puntaje & Categoría & Puntaje & Categoría \\
\hline Agudeza visual dinámica & +2 & Alterado & 1 & Normal & 0 & Normal \\
\hline Apoyo monopodal & $3 s$ & Alterado & $3 s$ & Alterado & $4 \mathrm{~s}$ & Alt. leve \\
\hline Alcalce funcional & $11 \mathrm{~cm}$ & Alterado & $22 \mathrm{~cm}$ & Normal & $30 \mathrm{~cm}$ & Normal \\
\hline Timed up and go & $39 \mathrm{~s}$ & Alterado & $18 \mathrm{~s}$ & Alterado & $9 \mathrm{~s}$ & Normal \\
\hline \multicolumn{7}{|l|}{ Dynamic gait index } \\
\hline - Marcha normal & 1 & Dificultad marcada & 2 & Dificultad leve & 3 & Normal \\
\hline $\begin{array}{l}\text { - Marcha con cambios } \\
\text { de velocidad }\end{array}$ & 0 & $\begin{array}{l}\text { No puede } \\
\text { realizar }\end{array}$ & 1 & $\begin{array}{l}\text { Dificultad } \\
\text { marcada }\end{array}$ & 3 & Normal \\
\hline $\begin{array}{l}\text { - Marcha con movimientos } \\
\text { de cabeza plano horizontal }\end{array}$ & 0 & $\begin{array}{l}\text { No puede } \\
\text { realizar }\end{array}$ & 2 & $\begin{array}{l}\text { Dificultad } \\
\text { leve }\end{array}$ & 3 & Normal \\
\hline $\begin{array}{l}\text { - Marcha con movimientos } \\
\text { de cabeza plano vertical }\end{array}$ & 0 & $\begin{array}{l}\text { No puede } \\
\text { realizar }\end{array}$ & 1 & $\begin{array}{l}\text { Dificultad } \\
\text { marcada }\end{array}$ & 3 & Normal \\
\hline
\end{tabular}

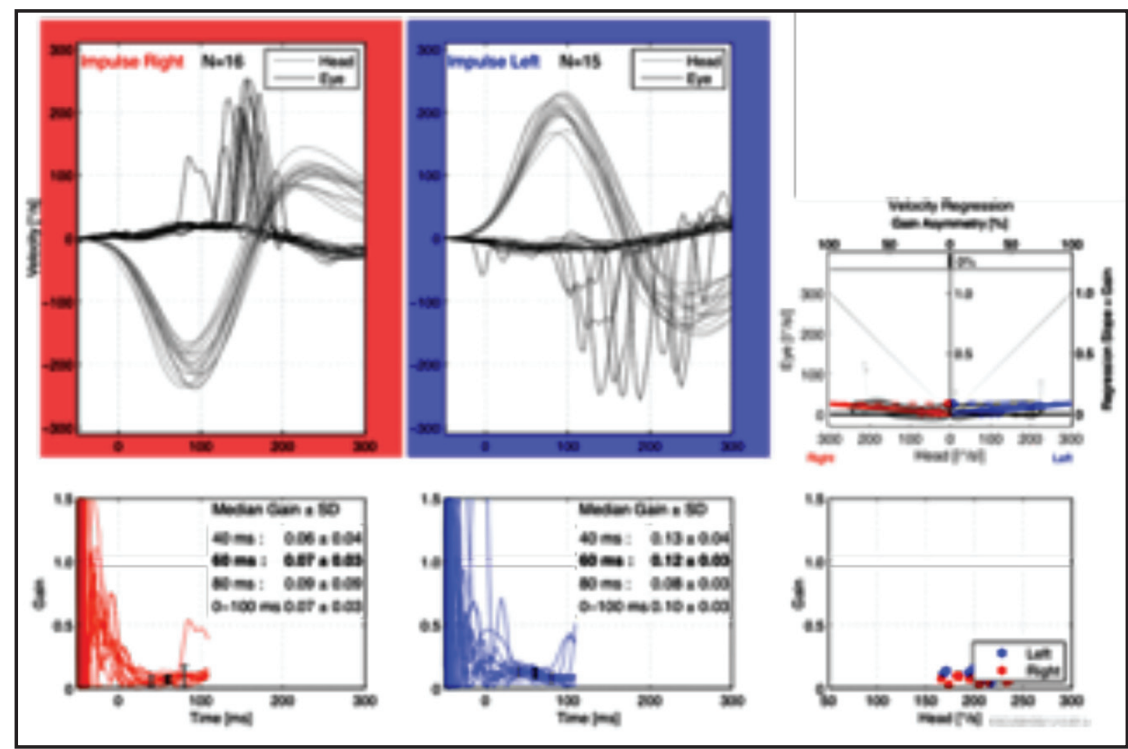

Figura 5. V-HIT plano horizontal realizado en evaluación intermedia. Se aprecia ganancia de 0.08 para oído derecho y 0.09 para oído izquierdo. Asimetría de $0 \%$. 


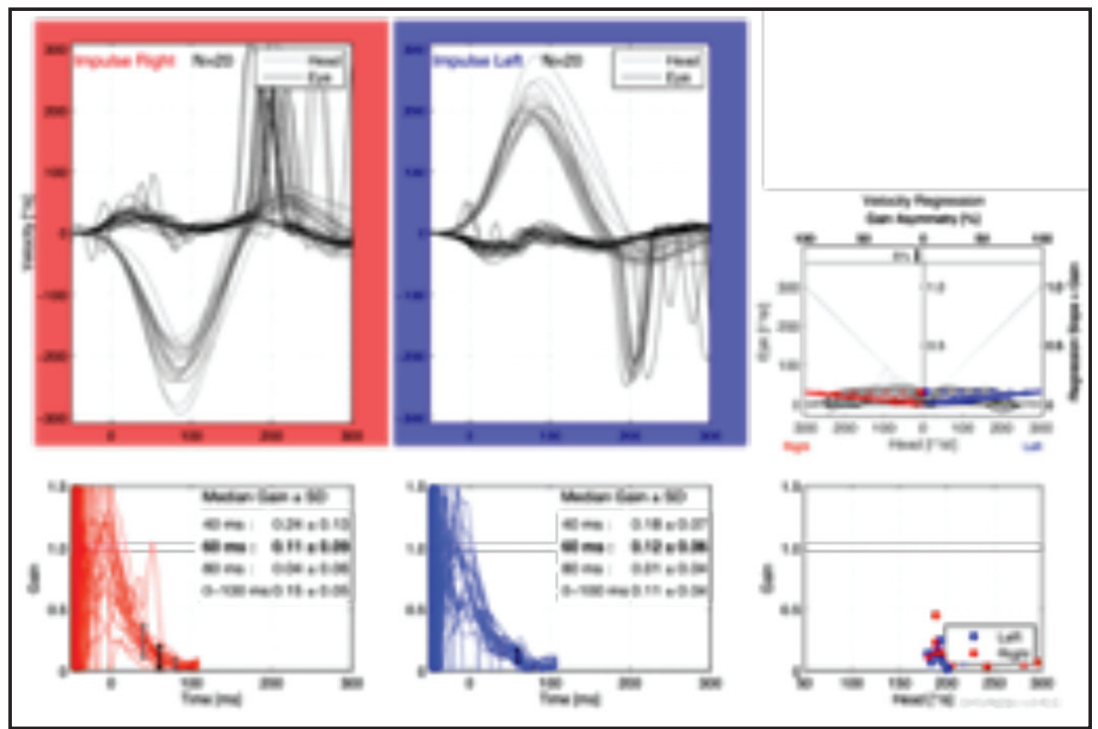

Figura 6. V-HIT plano horizontal realizado en evaluación final. Se aprecia ganancia de 0.10 para ambos oídos.

por la paciente en la evaluación inicial, lo cual culmina de acuerdo a evaluación final sin alteración. Por otra parte, en FRT se aprecia un disminuido alcance funcional en la evaluación previa, progresando significativamente en la evaluación intermedia y final. El test apoyo monopodal evidencia un avance limitado desde categoría "alterado" a "alteración leve".

EI DGI demuestra un progreso notorio en la marcha utilizando diversos tiempos y modalidades, de no poder realizar las pruebas o presentar marcada dificultad, a obtener un puntaje normal en todos los ítemes evaluados: marcha normal, con cambios de velocidad y movimientos de cabeza en planos horizontal y vertical. En este mismo sentido TUG evidencia un avance importante y progresivo respecto al tiempo que demora en recorrer un trayecto determinado. Finalmente, la prueba de V-HIT exhibe un reducido incremento de la ganancia del RVO horizontal bilateral entre las evaluaciones inicial, intermedia y final del programa. Las sacadas correctivas covert tienden a disminuir hacia la evaluación final, mientras que las sacadas overt se mantienen, aunque en patrones más organizados.

\section{DISCUSIÓN}

La ototoxicidad es el efecto nocivo, iatrogénico, con lesiones reversibles 0 permanentes de diversas sustancias sobre el oído, afectando la función coclear $y / 0$ vestibular ${ }^{16}$. Dentro de los aminoglucósidos, la gentamicina presenta especial selectividad por el laberinto posterior, afectando principalmente el componente vestibular del oído interno ${ }^{17}$. En este sentido, es importante mantener una actualización clínica respecto de los efectos adversos de múltiples medicamentos por las graves consecuencias que pueden generar a nivel otológico y en la calidad de vida de las personas, asimismo, establecer un seguimiento riguroso en los casos que la administración de dicho fármaco sea requerida.

La BVP se caracteriza principalmente por desequilibrio, oscilopsia, inestabilidad de la marcha con mayor dificultad en terrenos irregulares 0 en la oscuridad, así como también, ganancia del RVO reducida de manera bilateral registrada por medio de V-HIT, prueba calórica, bobina escleral y/o silla rotatoria ${ }^{2,5}$. Existe evidencia que demuestra que estos hallazgos permanecen en el tiempo, no encontrándose mejoría en los pacientes con 
BVP18, lo cual genera un importante impacto en la calidad de vida de las personas que la padecen, especialmente en las actividades de la vida diaria, aspectos emocionales y sociales $^{19}$, por lo que el abordaje de un equipo multidisciplinario es fundamental.

La terapia vestibular ofrece beneficios claros y sustanciales a los pacientes con BVP, evidenciándose una mejora en la función y disminución considerable de los síntomas ${ }^{13}$. Se ha visto que luego de un programa de ejercicios de sustitución y adaptación vestibular realizado semanalmente a 13 personas con BVP, sumado a un programa de ejercicios domiciliarios 4 a 5 veces por día (20 a 40 minutos en total) ${ }^{14}$, permiten una mejora en la agudeza visual dinámica respecto al grupo control. Por otra parte, un programa enfocado en ejercicios de equilibrio, marcha y estabilidad de la mirada demostró una progresión de los aspectos abordados, tras una intervención realizada por ocho semanas, acompañada con ejercicios diarios en el hogar (1 a 2 veces al día $)^{15}$.

En nuestra opinión, la integración de los ejercicios de marcha utilizando diversas modalidades en conjunto con movimientos cefálicos en plano horizontal y vertical, contribuyeron mayormente al éxito de la terapia. Sin embargo, consideramos que es importante continuar con ejercicios enfocados en estimular el RVO sumado a adaptación vestibular, para disminuir los síntomas residuales

\section{BIBLIOGRAFÍA}

1. Ward BK, Agrawal Y, Hoffman hJ, Carey JP, Della Santina CC. Prevalence and impact of bilateral vestibular hypofunction: Results from the 2008 US National Health Interview Survey. JAMA Otolaryngol Head Neck Surg 2013; 139: 803-10.

2. Strupp M, KIm J, Murofushi $T$ y col. Bilateral vestibulopathy: Diagnostic criteria Consensus document of the Classification Committee of the Bárány Society. J Vestib Res 2017; 27: 177-89.

3. Strupp M, Jahn K, Brandt T. Another adverse effect of aspirin: bilateral vestibulopathy. J Neurol Neurosurg Psychiatry 2003; 74: 687-91.

4. Fischer CS, Bayer 0, Strupp M. Transient relacionados con la sensación de visión borrosa en algunas situaciones. Investigaciones más recientes afirman que la RV mejora el equilibrio y la calidad de vida de las personas, también disminuye el mareo y riesgo de caídas ${ }^{20}$. Los resultados de un estudio que evaluó la efectividad de la RV personalizada y supervisada en sujetos con disfunción unilateral o bilateral fueron estadísticamente significativos de acuerdo a DHI, TUG, DGI, posterior a ocho semanas de tratamiento ${ }^{19}$, lo que indicaría mejoras en la discapacidad que genera la patología en el aspecto emocional y actividades de la vida diaria, así como en la estabilidad postural y equilibrio dinámico.

\section{CONCLUSIÓN}

El uso de gentamicina puede provocar daños irreversibles en la función vestibular. La BVP es poco frecuente y afecta considerablemente la calidad de vida de las personas que la padecen, específicamente los aspectos físico, emocional, funcional y social. La RV ofrece beneficios sustanciales en el control postural, la mantención del equilibrio y la marcha, así como también en las actividades de la vida diaria. El trabajo multidisciplinario es fundamental en el diagnóstico y abordaje de estos pacientes, pues permite brindar un pronóstico favorable en su recuperación.

bilateral vestibular dysfunction caused by intoxication with low doses of styrene. Eur Arch Otorhinolaryngol 2014; 271: 619-23.

5. Strupp M, Fell K, Dieterich M, Brandt T. Bilateral vestibulopathy. Handb Clin Neurol 2016; 137: 235-40.

6. Lucieer F, Vonk P, Guinand N, Stokroos R, Kingma H, VAN DE BERG R. Bilateral vestibular hypofunction: insights in etiologies, clinical subtypes, and diagnostics. Front Neurol 2016; 7: 26.

7. Huang R, Bı Gr. Bilateral vestibulopathy. J Clin Otorhinolaryngol Head Neck Surg 2017; 31: 1937-9.

8. LIN $Y, G A O L X, L I L$ Y coLs. Etiology analysis and vestibular assessment of bilateral vestibular vestibulopathy. J Clin Otorhinolaryngol Head Neck Surg 2018; 32: 379-82. 
9. Strupp M, Mandalà M, López-Escámez J. A Peripheral vestibular disorders. Current Opinion in Neurology 2019; 32: 165-73.

10. Hermann R, Ionescu E, Dumas 0, Trigali S, Truy E, TILIKETE C. Bilateral Vestibulopathy: Vestibular Function, Dynamic Visual Acuity and Functional Impact. Front Neurol 2018; 9: 1-10.

11. Van de Berg R, van Tilburg M, Kingma $H$. Bilateral vestibular hypofunction: challenges in establishing the diagnosis in adults. ORL J Otorhinolaryngol Relat Spec 2015; 77: 197218.

12. Fusimoto $\mathrm{C}, \mathrm{Yagl}_{\mathrm{A}} \mathrm{M}$, Murofushi T. Recent advances in idiopathic bilateral vestibulopathy: a literature review. Orphanet Journal of Rare Diseases 2019; 14: 202.

13. Hall C, Herdman S, Whitney S. Vestibular Rehabilitation for Peripheral Vestibular Hypofunction: An Evidence-Based Clinical Practice Guideline: From the American Physical Therapy Association Neurology section. J Neurol Phys Ther 2016; 40: 124-55.

14. Herdman SJ, Hall CD, Schubert MC, Das VE, Tusa RJ. Recovery of dynamic visual acuity in bilateral vestibular hypofunction. Arch Otolaryngol Head Neck Surg 2007; 133: 383-9.

15. Krebs DE, Gill-Body KM, Parker SW, Ramirez JV, Wernick-Robinson M. Vestibular rehabilitation: useful but not universally. Otolaryngol Head Neck Surg 2003; 128: 240-50.

16. Quintero J, Hernández M, de León N, Meléndez L. Ototoxicidad y factores predisponentes. Rev Cubana de Pediatr 2018; 90: 111-31.

17. Ishiyama $G$, Ishiyama $A$, Kerber $K$, Baloh R. Gentamicin ototoxicity: clinical features and the effect on the human vestibulo-ocular réflex. Acta Otolaryngol 2016; 126: 1057-61.

18. Zingler VC, Weintz E, Jahn K y col. Follow-up of vestibular function in bilateral vestibulopathy. $J$ Neurol Neurosurg Psychiatry 2008; 79: 284-8.

19. Karapolat H, Celebisoy N, Kirazli Y y col. Is vestibular rehabilitation as effective in bilateral vestibular dysfunction as in unilateral vestibular dysfunction? Eur J Phys Rehabil Med 2014; 50 : 657-63.

20. Alghadir AH, Iqbal ZA, Whitney SL. An update on vestibular physical therapy. J Chin Med Assoc 2013; 76: 1-8. 